\title{
Short-term variation of central corneal thickness measurements in keratoconus using the Tono- Pachymeter NT530P (Tonopachy ${ }^{\text {TM}}$ )
}

\author{
KP Mashigea, OA Oduntanb and NM Gcabashe
}

a, b, c Discipline of Optometry, School of Health Sciences, University of KwaZulu-Natal, Westville Campus, Private Bag X54001, Durban, 4000 South Africa

<mashigek@ukzn.ac.za>

Received 13 March 2012; revised version accepted 31 August 2012

\begin{abstract}
Repeatability of values is a crucial reliability index in any instrument used to measure ocular parameters. The purpose of this study was to investigate short-term variability of central corneal thickness (CCT) measurements in keratoconic eyes. The Tono-Pachymeter NT530P (Tonopachy ${ }^{\mathrm{TM}}$ ) was used to take fifty successive automatic measurements of CCT of the right eyes of ten keratoconic subjects (six females and four males), aged 19 to 41 years (mean $=30.4 \pm 6.8$ years). The means, standard deviations and variances of the measurements showed little variation with good repeatability. The
\end{abstract}

inter-subject or overall mean values and standard deviations of the measurements were $452.1 \pm 30.3$ $\mu \mathrm{m}$. The Kolmogorov-Smirnov (K-S), Lilliefors and Shapiro-Wilks (S-W) tests showed that generally the data was normally distributed, with a few exceptions. The results of this study suggest that over short periods of time, the Tonopachy ${ }^{\mathrm{TM}}$ provides repeatable measures of CCT in keratoconic corneas. (S Afr Optom 2012 71(3) 102-108)

Key words: Tonopachy ${ }^{\mathrm{TM}}$, central corneal thickness, keratoconus, repeatability, univariate normality.

\section{Introduction}

Keratoconus is a disease of unknown etiology which is characterized by thinning and protrusion of the central or paracentral cornea ${ }^{1,2}$. This corneal thinning can be measured easily in humans by pachymetry and this measurement is used for diagnosis and management of keratoconus and other ectasias. Corneal thickness measurement in the human eye is also important in monitoring and planning of corneal graft ${ }^{3}$, accurately measuring eye pressure ${ }^{4-7}$, and as an indicator of corneal tissue hydration in the eye ${ }^{8}$. Corneal thickness can be evaluated with a variety of optical or ultrasound based methods such as Scheimpflug imaging, ultrasound and optical coherence tomography 9,10 . One of the most important issues relates to whether the measurements by an instrument are valid, reliable and repeatable. Over the years, several studies ${ }^{10-13}$ have investigated the reliability and repeatability of various pachymeters. Such studies have shown that pachymeters provide reasonably accurate and repeatable measures of corneal thickness in normal subjects ${ }^{10-13}$.

Although ultrasound pachymetry is often regarded as the gold standard for measurement of corneal thickness

\footnotetext{
a BSc BOptom CAS MOptom

b BSc(Hons)Optom PhD

${ }^{\mathrm{c}}$ BOptom
} 
due to its high degree of intra-examiner, inter-examiner and inter-instrument reproducibility ${ }^{10-14}$ the procedure is invasive and shows "variability of measurements caused by probe misalignment or decentering and changes in the speed of sound in corneal tissues with different degrees of hydration"14. Several non-contact methods have been introduced in order to overcome the limitations of ultrasound pachymetry. Also, some studies ${ }^{15,16}$ have examined the repeatability and agreement of several non-contact methods for the measurement of CCT in normal and post LASIK subjects. Among these, the Tonopachy ${ }^{\mathrm{TM}}$ (Nidek, Japan) was introduced recently which simultaneously measures CCT and IOP values. A recent study ${ }^{17}$ compared the CCT values measured with Tonopachy ${ }^{\mathrm{TM}}$ to those measured with Pentacam and ultrasound pachymeters. The results showed that values from ultrasound pachymetry were lowest (mean $=541.7 \pm 30.6 \mu \mathrm{m})$ whereas those by Tonopachy ${ }^{\mathrm{TM}}$ and Pentacam were similar (means $=557.3 \pm 34.3$ and $558.0 \pm 33.7 \mu \mathrm{m}$ respectively). Tonopachy ${ }^{\mathrm{TM}}$ overestimated CCT by $13.9 \mu \mathrm{m}$ when compared with those of ultrasound pachymetry in 104 subjects with normal corneas ${ }^{17}$. The authors ${ }^{17}$ concluded that Tonopachy ${ }^{\mathrm{TM}}$ is a reliable instrument for evaluating CCT, however, careful attention should be paid when comparing Tonopachy ${ }^{\mathrm{TM}}$ CCT measurement with ultrasound pachymetry as the values may not be interchangeable. Lomoriello et al18 and Mashige et $a l^{19}$ reported that the Tonopachy ${ }^{\mathrm{TM}}$ provides accurate and reliable CCT measurements in healthy corneas. However, its reliability in assessing CCT in eyes with corneal diseases such as keratoconus has not been established. The purpose of this study therefore, was to evaluate short-term variability in CCT measurements in keratoconic eyes using the Tono-Pachymeter NT530P (Tonopachy ${ }^{\mathrm{TM}}$ ).

\section{Methods}

The study was performed in accordance with the ethical standards stated in the Declaration of Helsinki and the protocol was approved by the Research and Ethics Committee of the University of KwaZuluNatal. Written informed consent was obtained from all the participants after the nature and intent of the study had been fully explained to them. Ten subjects with keratoconus (varying from early to advanced keratoconus) as verified with refraction, keratometry, characteristic topographic patterns and slit-lamp examination participated in the study. The Bausch and Lomb keratometer ${ }^{\mathrm{TM}}$ has a range ${ }^{20}$ of $36.00 \mathrm{D}$ to 52.00 $\mathrm{D}$, measured in increments of $0.12 \mathrm{D}$. The advanced form of keratoconus $(>52.00 \mathrm{D})$ exceeded the keratometer's measuring ability. Therefore, $\mathrm{a}+1.25 \mathrm{D}$ lens was placed over the keratometer aperture to extend ${ }^{20}$ the range by approximately $9.00 \mathrm{D}$. Slit-lamp findings included one or more of the following signs: conical protrusion, Fleischer's ring, Vogt's striae or anterior stromal thinning. All subjects reported excellent general health and apart from keratoconus, none of participants had any other ocular abnormalities, history of eye disease or prior refractive surgery. To minimize possible contact lens-induced swelling, the participants were instructed not to wear their contact lenses from the evening before the data collection day until after the data collection.

Fifty CCT automatic measurements of the right eye of each subject were taken by one examiner between $12 \mathrm{pm}$ and $2 \mathrm{pm}$. The measurements were obtained in approximately 10 to 15 minutes for each eye due to the auto-alignment function of the instrument and the measurements were printed out for recording. The testing room was set up in one of the Optometry clinics at the University of KwaZulu-Natal and all the tests were performed by the same operator with the eyes in an undilated state.

The Statistica Ver ${ }^{8}$ software programme was used for all statistical analysis. The distributions of the CCT measurements were plotted using box and whisker plots, histograms and normal probability plots. The ranges, means and standard deviations were determined. The Kolmogorov-Smirnov (K-S), Lilliefors and the ShapiroWilks (S-W) tests were done to explore univariate normality of the data and $p$-values were obtained to determine whether samples were normally distributed and whether parametric statistical tests were appropriate.

\section{Results}

The subjects included seven Indians and three Blacks, aged between 19 and 41 years (mean $=30.4 \pm 6.8$ years). The mean keratometric values obtained with the Bausch and Lomb keratometer ranged from 47 to $61 \mathrm{D}$ (mean $=50 \pm 3.50 \mathrm{D})$ and corneal astigmatism ranged from 3.00 to $7.50 \mathrm{D}($ mean $=4.50 \pm 1.50 \mathrm{D})$. Table 1 shows the descriptive statistics such as means, standard deviations minimum and maximum values for the CCT 
Table 1: Means, standard deviations, maxima and minima for CCT measurements in micrometres ( $\mu \mathrm{m})$ for the 10 subjects are given. A negative sign indicates negative skewing or platykurtosis. The last two columns are unit-less.

$\begin{array}{lrrcccc}\text { Subject } & \text { Mean } & \text { Std. Deviation } & \text { Minimum } & \text { Maximum } & \text { Skewness } & \text { Kurtosis } \\ 1 & 490 & 2.8 & 485 & 499 & 0.8 & 0.9 \\ 2 & 424 & 3.1 & 419 & 433 & 0.6 & 0.5 \\ 3 & 432.3 & 4.0 & 425 & 444 & 0.7 & 1.0 \\ 4 & 451.8 & 2.8 & 447 & 457 & 0.1 & -1.2 \\ 5 & 489.5 & 2.5 & 484 & 495 & -0.2 & -0.6 \\ 6 & 482.1 & 2.5 & 475 & 487 & -0.6 & 1.0 \\ 7 & 411.7 & 5.7 & 401 & 426 & 0.4 & -0.3 \\ 8 & 432 & 2.0 & 428 & 436 & -0.3 & 0.0 \\ 9 & 421.2 & 2.7 & 413 & 430 & 0.1 & 2.8 \\ 10 & 486 & 2.5 & 481 & 492 & 0.3 & -0.2 \\ \text { Total } & 452.1 & 30.3 & 401 & 499 & 0.1 & -1.7\end{array}$

Figure 1 shows the box and whisker plots for the CCT $(\mu \mathrm{m})$ values of the right eyes of the 10 subjects. The CCT means (see Table 1) are indicated by lines through the middle of the box part of the box and whisker plot while the ends of the box depict the standard deviations of the distribution. The whiskers represent the minimum and maximum values for each subject.

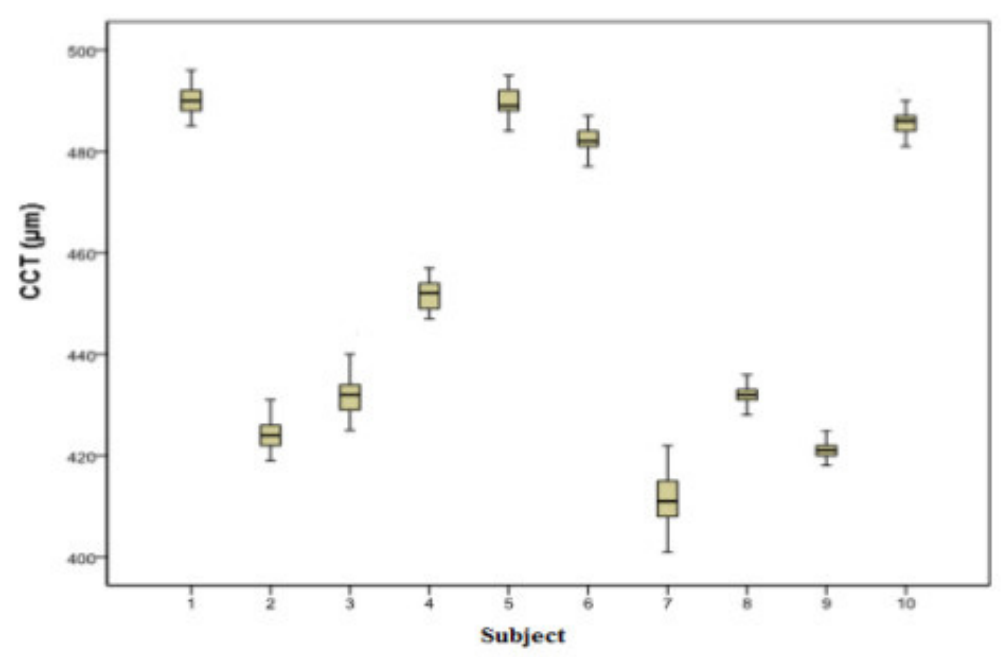

Figure 1: The box and whisker plots for CCT measurements in micrometers ( $y$-axis) for the right eyes of the 10 subjects.

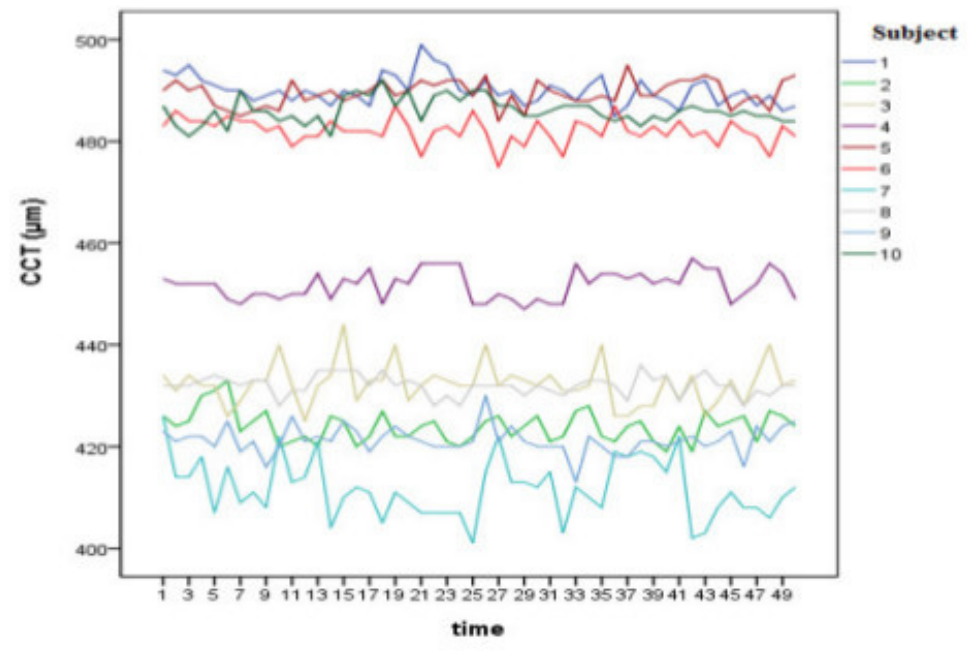

Figure 2: Profiles of short-term variation of the 50 measurements of the CCT for 10 subjects are indicated. The CCT profile of subject 7 (bottom light blue colour) appears to be more variable (401 to 426 $\mu \mathrm{m})$ than the others.

The temporal profiles for the samples of CCT values for all subjects ( $N=50$ per sample) are shown in Figure 2 to allow the reader to inspect the repeatability and variation of the measurements of each subject. Some but not all of the profiles appear relatively flat.

The CCT samples for all participants except subjects 3 and 8 were normally distributed. Figures 3 and 4 show histograms and normal probability plots for the samples of CCT for subjects 5 and 6 , which were randomly selected from the eight CCT samples that were normally distributed. Normality of the samples was also tested using the K-S, Lilliefors and S-W tests (see the relevant figure captions for such results). 
a)

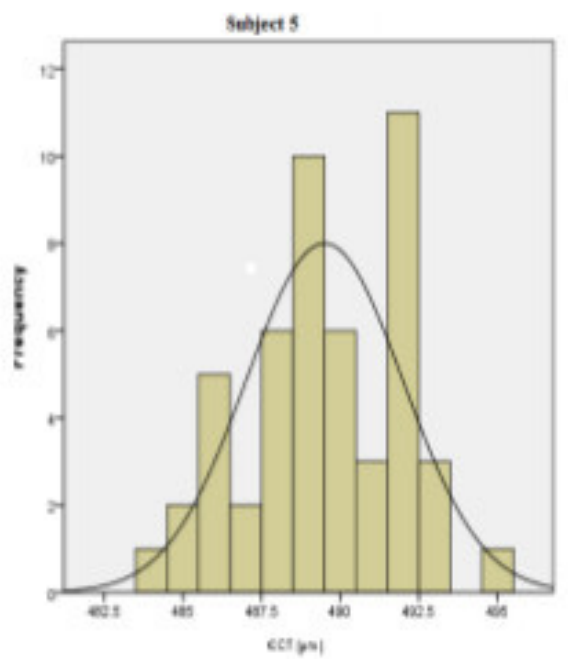

b)

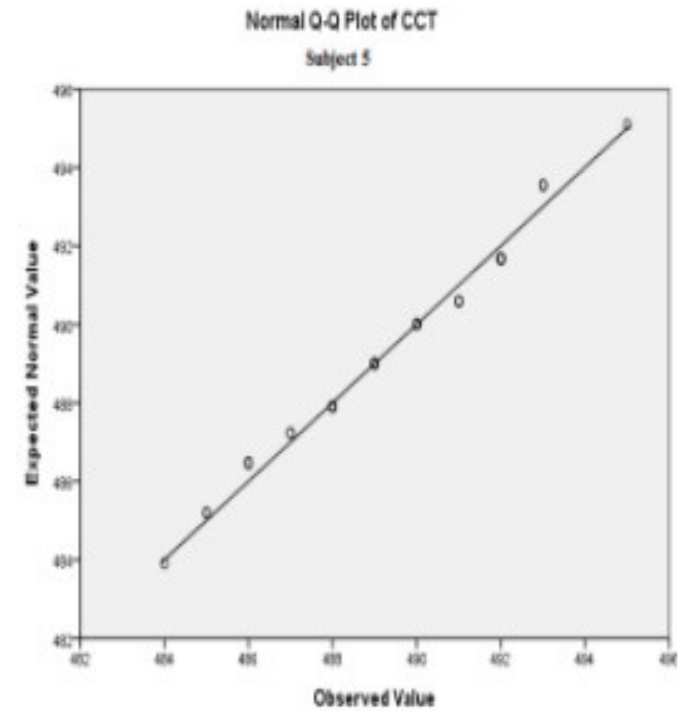

Figure 3: Histograms (a) and normal probability (b) plots for the CCT sample of subject 5 . The samples were normally distributed (the $p$-values for the K-S, Lilliefors and S-W tests were all greater than 0.05 ).
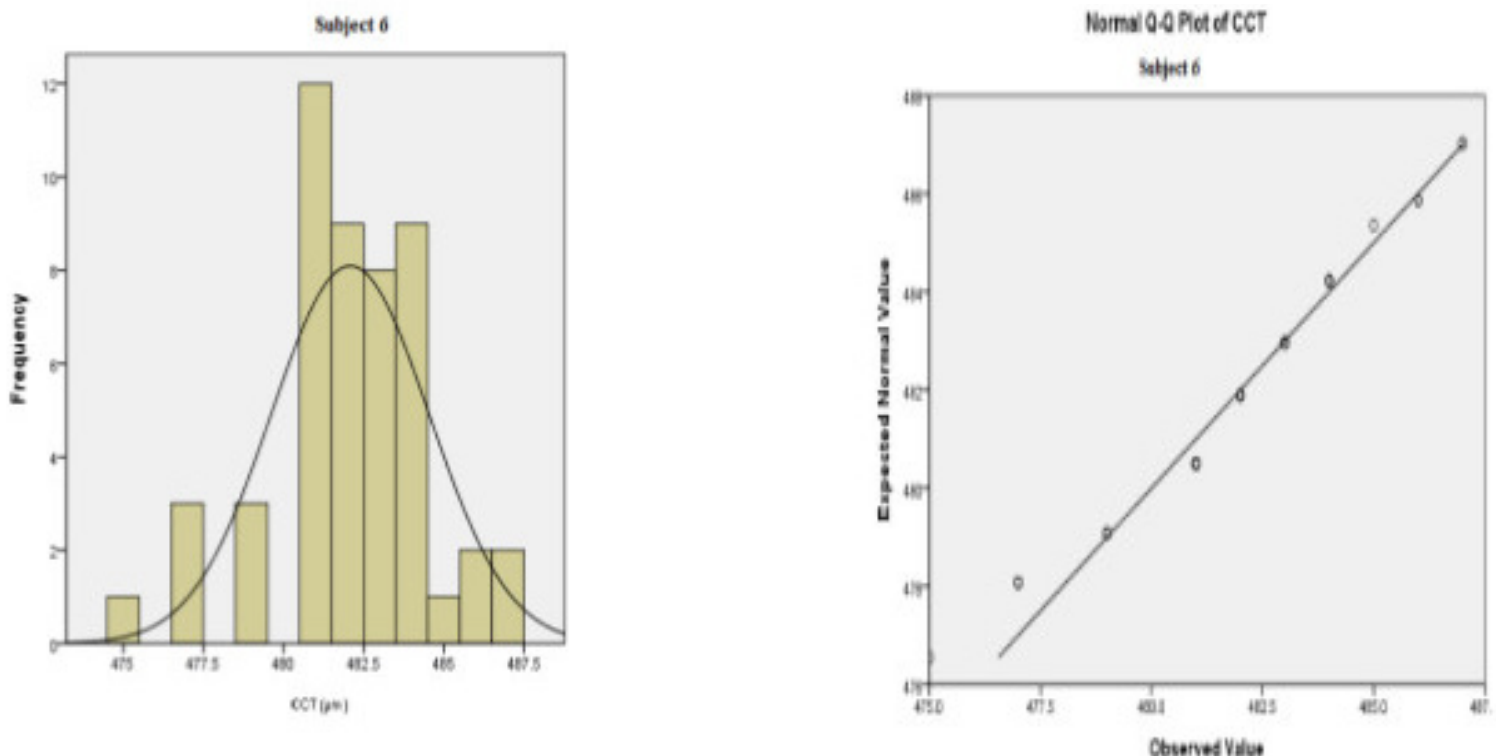

Figure 4: Histograms and normal probability plots for subject 6. The histogram shows the frequency distribution for the CCT measurements.
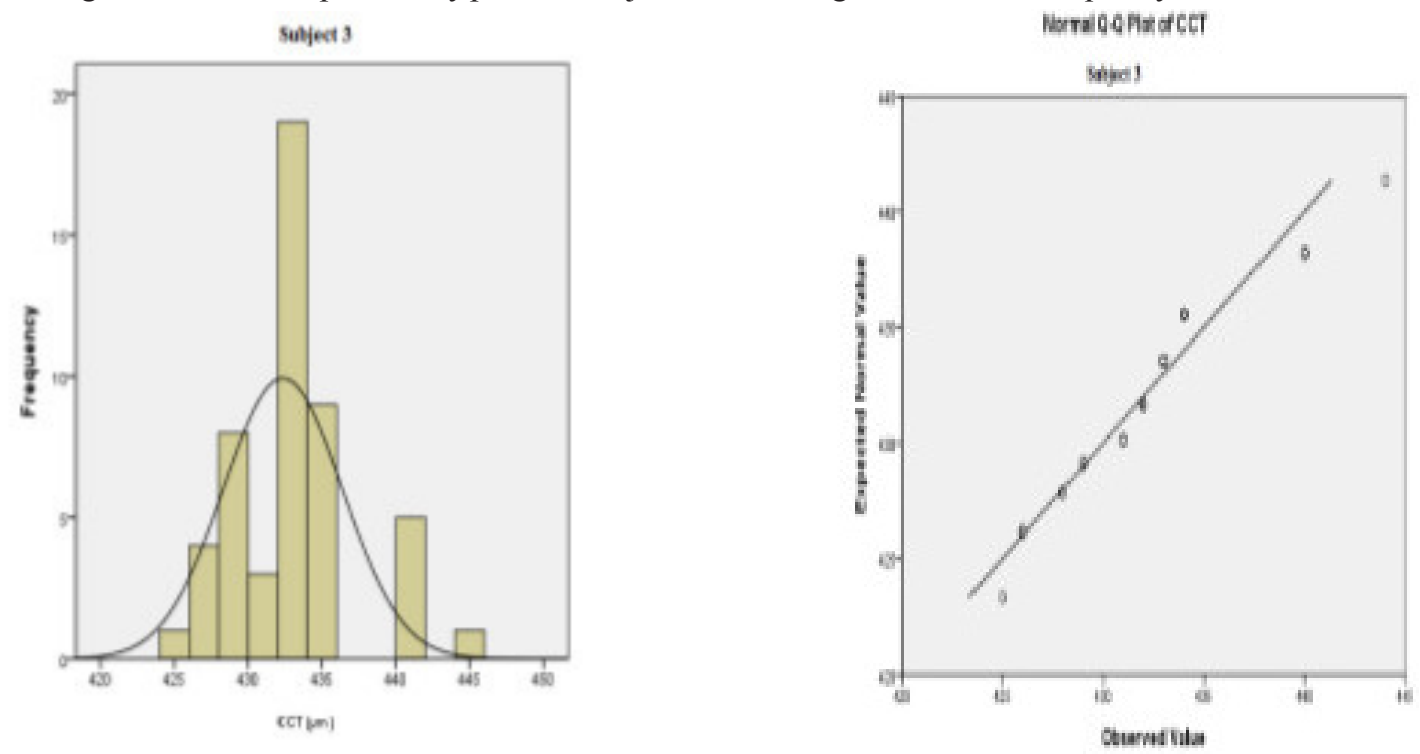

Figure 5: Histograms and normal probability plots for the CCT sample of subject 3. The sample for CCT was not normally distributed (the $p$-values for the K-S, Lilliefors and $\mathrm{S}-\mathrm{W}$ tests were $<0.05$ ). 

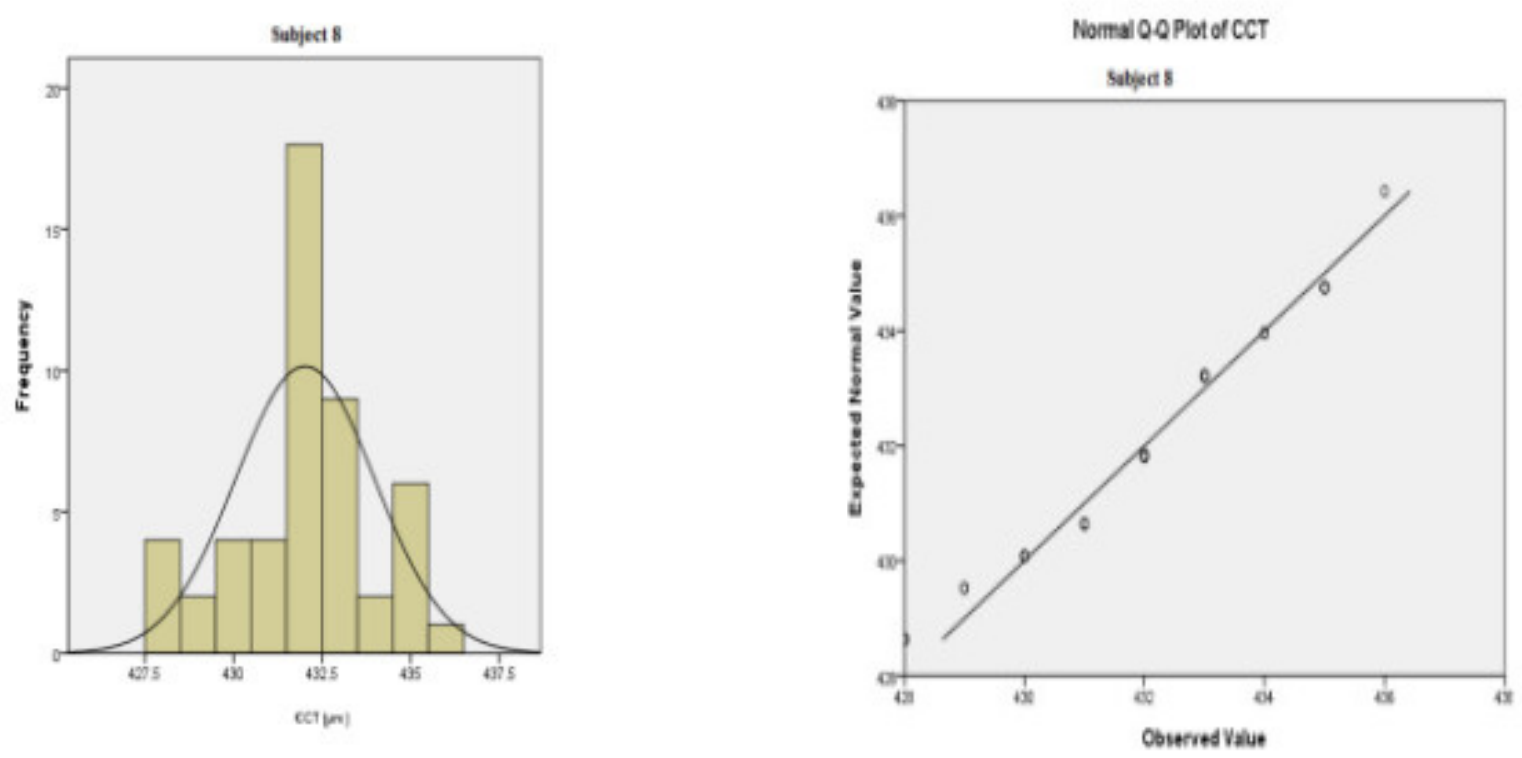

Figure 6: Histogram and normal probability plot for the CCT of subject 8 . The samples were not normally distributed according to the $p$-values for the K-S, Lilliefors and S-W tests. The $p$-values for all the three tests were $<0.05$, suggesting rejection of the null hypothesis that the sample is from a normally distributed population.

The data for subjects 3 and 8 demonstrated departure from normality. Figures 5 and 6 include histograms and normal probability plot for subjects 3 and 8 that demonstrated departure from normality for the CCT.

\section{Discussion}

The measurement of CCT is useful in identifying corneal thinning disorders such as keratoconus ${ }^{1,}$. Ultrasound biometry has been the most common method for measuring $\mathrm{CCT}^{10-13}$. A major advantage of ultrasound biometry is that it requires minimal observer judgment and is therefore consistent and repeatable between observers ${ }^{10-13}$. However, the use of a topical anesthetic agent, its invasive nature (which may increase the risk for infection), exact alignment at the centre of the cornea and proper handling of the probe perpendicular to the corneal surface are some of the limitations of this technique ${ }^{10-13}$. It is also not advised to rely exclusively on ultrasonic pachymetry to exclude or diagnose keratoconus ${ }^{10-13}$. These potential limitations stimulated the development of possibly more reliable, repeatable and operator independent non-contact ocular biometry techniques including the Tonopachy ${ }^{\mathrm{TM}}$, Pentacam, Orbscan, optical coherence tomography and other optical methods for imaging and measuring the $\mathrm{CCT}^{10}$. This study is the first to investigate shortterm variation of CCT in keratoconic eyes using the

\section{Tonopachy ${ }^{\mathrm{TM}}$.}

Although the sample size in this study was not as large as that used in recent studies ${ }^{18,19}$ with the Tonopachy ${ }^{\mathrm{TM}}$ it was similar to that of previous studies with Scheimpflug photography via the Oculus Pentacam ${ }^{10}$. However, the 50 consecutive measurements taken and analysed per eye seemed sufficient to determine the short-term variation of the Tonopachy ${ }^{\mathrm{TM}}$ in keratoconic eyes. The measured keratometric and CCT values indicate that the eyes studied had keratoconus that varied from moderate to severe ${ }^{1}$, therefore covering a wide range of corneal thicknesses.

In cases of keratoconus, several devices are used to assess the CCT and the repeatability of the measurements is very important for diagnosis of the disease and monitoring of its progression. The Tonopachy ${ }^{\mathrm{TM}}$ allows fast, non-contact measurement of the CCT of the individual while providing good comfort and avoiding application of topical anesthesia ${ }^{18,21}$. The instrument provides the CCT via Scheimpflug camera principles ${ }^{18,21}$. An additional advantage is that this instrument also provides automatic and simultaneous measurements of the individual's intra-ocular pressure $18,21$.

The box and whisker plots for the CCT (see Figure 1) showed that the standard deviations were small suggesting that multiple measurements of CCT in keratoconus with the Tonopachy ${ }^{\mathrm{TM}}$ were similar for 
each of the subjects. The line plots of the short-term variation shown in Figure 2 are also mainly relatively flat, further suggesting that there is minimal variation in CCT measurements of the keratoconic eyes. Therefore, the CCT measured with Tonopachy ${ }^{\mathrm{TM}}$ are largely repeatable within individuals. This suggests that the instrument is a potentially useful clinical and researchoriented device. However, some CCT profiles were more variable than others. For instance, CCT of subject 7 ranged from 401 to $426 \mu \mathrm{m}$ with a mean of $411.7 \mu \mathrm{m}$ and a standard deviation of $5.7 \mu \mathrm{m}$. This subject had severe keratoconus $(61 \mathrm{D})$. It is therefore possible that variations in CCT in the short-term may occur in severe keratoconus. Further investigation is recommended wherein repeated $\mathrm{CCT}$ measures are undertaken on multiple subjects with severe keratoconus. All samples were also investigated for normality using the K-S, Lilliefors and S-W tests. Probabilities were used in determining whether these statistics were significant and whether there was departure from univariate normality. Probabilities were greater than 0.05 for most, but not all, indicating that most samples were essentially normal distributions ${ }^{22}$.

Positive values for the skewness indicate data that are positively skewed towards larger values and negative values for the skewness indicate data that are negatively skewed. Similarly, positive values for kurtosis indicate leptokurtosis and a negative value indicates platykurtosis. As shown in Table 1 , in the 10 eyes studied, CCT measurements showed minimal variation. No sample showed profound skewness and only subject 9 showed some leptokurtosis (2.8) while subject 4 demonstrated a statistical measure that indicates a distribution that has some platykurtosis $(-1.2)$.

In conclusion, Tonopachy ${ }^{\mathrm{TM}}$ provides measurements of CCT that are repeatable and it is therefore suitable to aid in the diagnosis and monitor the progression of keratoconus when measurements are repeated over a short time. A possible limitation of this study is the limited number of cases and therefore, future studies including repeated measures on a large population sample are recommended.

\section{References}

1. Koenig SB. Bilateral recurrent self-induced keratoconus. Eye \& Contact Lens 200834 343-344.

2. Klyce SD. Chasing the suspect: keratoconus. Br J Ophthalmol 200993 845-847.

3. Zadnik K, Barr JT, Gordon MO, Edrington TB. Biomicroscopic signs and disease severity in keratoconus. Collaborative Longitudinal Evaluation of Keratoconus (CLEK) Study Group. Cornea 199615 139-146.

4. Tonnu PA, Ho T, Newson T, El Sheikh A, Sharma K, White E, Bunce C, Garway-Heath D. The influence of central corneal thickness and age on intraocular pressure measured by pneumotonometry, non-contact tonometry, the Tono-Pen XL and Goldmann applanation tonometry. Br J Ophthalmol 200589 851-854.

5. Sahin A, Basmak H, Yildirim N. The influence of central corneal thickness and corneal curvature on intraocular pressure measured by Tono-Pen and Rebound tonometer in children. J Glaucoma 200817 57-61.

6. Shafiq I. Influence of central corneal thickness (CCT) on intraocular pressure (IOP) measured with Goldmann applanation tonometer (GAT) in normal individuals. Pak J Ophthalmol 200824 196-200.

7. Gelaw Y, Kollman M, Irungu NM, Ilako DR. The influence of central corneal thickness on intraocular pressure measured by Goldmann applanation tonometry among selected Ethiopian communities. J Glaucoma 201019 514-518.

8. Doughty MJ, Jonuscheit S. The Orbscan acoustic (correction) factor for central corneal thickness measures of normal human corneas. Eye \& Contact Lens 20102 106-115.

9. Swartz T, Marten L, Wang M. Measuring the cornea: the latest developments in corneal topography. Curr Opin Ophthalmol 200718 325-333.

10. Mathebula SD, Rubin A. Short-term variation of central corneal thickness and axial anterior chamber depth of healthy eyes using Scheimpflug photography via the Oculus Pentacam. S Afr Optom 200968 12-14.

11. Prospero Ponce CM, Rocha KN, Smith SD, Krueger RR. Central and peripheral corneal thickness measured with optical coherence tomography, Scheimpflug imaging, and ultrasound pachymetry in normal, keratoconus-suspect, and post-laser in situ keratomileusis eyes. $J$ Cataract Refract Surg 200935 1055-1062.

12. Buehl W, Stojanac D, Sacu S, Drexler W, Findl O. Comparison of three methods of measuring corneal thickness and anterior chamber depth. Am J Ophthalmol 2006 141 7-12.

13. Amano S, Honda N, Amano Y, Yamagami S, Miyai T, Samejima T, Ogata M, Miyata K. Comparison of central corneal thickness measurements by rotating Scheimpflug camera, ultrasonic pachymetry, and scanning-slit corneal topography. Ophthalmol $2006113937-941$.

14. De Santis U, Missolungi A, Mutani B, Richiardi L, Grignolo FM. Reproducibility and repeatability of central corneal thickness measurement in keratoconus using the rotating Scheimpflug camera and ultrasound pachymetry. Am J Ophthalmol 2007144 712-718. 
15. Huang J, Pesudovs K, Yu A, Wright T, Wen D, Li M, Yu Y, Wang Q. A comprehensive comparison of central corneal thickness measurement. Optom Vis Sci 201188 940-949.

16. Gorgun E, Yenerel NM, Dinc UA, Oncel B, Kucumen RB, Oral D, Ciftci F. Comparison of non-contact methods for the measurement of central corneal thickness. Ophthalmic Surg Lasers Imaging 201142 400-407.

17. Lee YG, Kim JH, Kim NR, Kim CY, Lee ES. Comparison between Tonopachy and other tonometric and pachymetric devices. Optom Vis Sci 201188 843-849.

18. Lomoriello DS, Lombardo M, Tranchina L, Oddone F, Serrao S, Ducoli P. Repeatability of intra-ocular pressure and central corneal thickness measurements provided by a noncontact method of tonometry and pachymetry. Graefes Arch Clin Exp Ophthalmol 2011249 429-434.

19. Mashige KP, Rampersad N, Jhetam S, Govender P. Short-term variation in central corneal thickness and intraocular pressure using the Tono-Pachymeter NT530P (Tonopachy ${ }^{\mathrm{TM}}$ ). S Afr Optom 201271 10-19.

20. Verma A. Optical management of keratoconus. Ind J Prac Doc 20085 11-12.

21. Kotecha A, Crabb DP, Spratt A, Garway-Heath DF. The relationship between diurnal variations in intraocular pressure measurements and central corneal thickness and corneal hysteresis. Invest Ophthalmol Vis Sci 200950 4229-4236.

22. Steinskog DG, Tjøstheim DB, Kvamstø NG. A cautionary note on the use of the Kolmogorov Smirnov test for normality. Mon Wea Rev 2007135 1127-1157. 